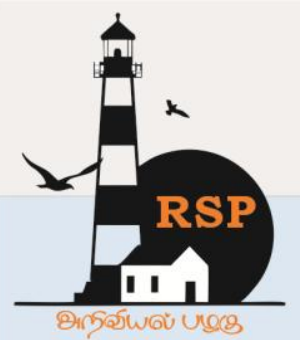

INTERNATIONAL RESEARCH JOURNAL ON

e-ISSN : 2582 - 4376 ADVANCED SCIENCE HUB Open Access

RSP SCIENCE HUB

(The Hub of Research Ideas)

Available online at www.rspsciencehub.com

\title{
Temperature based Fan Speed Controller
}

Srinivas $P^{1}$, Kavinkumar $B^{2}$, Arun Venkat $A^{3}$, Dr.R.Senthil Kumar ${ }^{4}$

${ }^{1,2,3}$ Student, Department of Electrical and Electronics Engineering, SNS College of Technology, Coimbatore-35.

${ }^{4}$ Associate professor, Department of Electrical and Electronics Engineering, SNS College of Technology, Coimbatore-35.

srinisrinivasp27@gmail.com ${ }^{1}$, Kavinbala2000@gmail.com ${ }^{2}$, ayiravenkat@gmail.com ${ }^{3}$ senkumarme@gmail.com ${ }^{4}$

Abstract

This report aims to give an overview of a standalone automatic fan speed controller that controls the speed of an electric fan according to our requirement. With the help of the embedded technology makes more efficient and reliable with this closed loop feedback control system. This project is the Temperature Based Fan Speed Control \& Monitoring with the help of the Arduino UNO and LM35 Temperature Sensor. In this the microcontroller controls the speed of an electric fan according to the requirement \& allows faster control and the LCD display makes the system user-friendly. The LCD panel displays the Sensed temperature in Celsius Scale and fan speed in percentage simultaneously.

\section{Keywords: Arduino, UNO, LM35 Temperature Sensor, Fan etc}

\section{Introduction}

The idea behind the project is to control the speed of the fan by difference in temperature. The Temperature variation in the fan is an different way to deal with the speed of the motor. It is a process in which the objects temperature is measured and the way of heat energy passes into or out of the object is correctly adjusted to achieve a stable temperature. This project attendances the design and simulation of the fan speed control system by using PWM technique based on the room temperature. How the room temperature can be measured? The answer to the simple question is with the help of a Temperature Sensor. It has been used to measure the temperature of the room and the speed of the fan is varied according to the room temperature using Pulse Width Modulation technique.

\subsection{Proposed System:}

In this project, microcontroller is most important and plays a vital role in the smart systems development. It has become an essential part in the current day to day technologies. This article discusses about the temperature based fan speed control an Arduino system. This system is responsible for control the cooling system automatically based on the room temperature. The system requires an Arduino board to implement a control system to this project. Since this is proposed to control the speed of the fan by varying the temperature with the help of the IOT technology.

\section{Descriptions:}

This project Temperature Based Fan Speed Control s can be done by using Arduino board with some electronics materials. The Arduino UNO board is very popular among all electronic circuits, 


\section{www.rspsciencehub.com}

thus we employed Arduino UNO board for the operation of the fan speed control. In the proposed system itself said that it is designed to detect the temperature of the room and send that information to the Arduino UNO board. Then the Arduino UNO board carries out the contrast of current temperature and set temperature based on the inbuilt program of the Arduino that feed through us. The output obtained from the operation is given through the o/p port of an Arduino UNO board to the LCD display that connected with the board. This generates the PWM pulses from the board which is further given to the driver circuit to get the expected output to the fan. [1-4]

\section{Components used:}

The components used are

1. Arduino UNO

2. Temperature Sensor

3. Fan

4. LCD Display

5. Potentiometer

6. Transistor

7. Resistor

8. Relay

9. Connecting wires

\subsection{Arduino UNO:}

Arduino UNO is a development board which contains microcontroller in the board itself. It is an open-source software. In the electronics platform, arduinois easy to use hardware and software. The Arduino boards can read inputs so that they can understand and give as some of the outcomes like light on a sensor, a finger on a button, activating a motor, turning on an LED, publishing something manually in online etc, that are all given as output to us. The most of the applications on everyday life, ardunio is a part of the application. The reason is that the Arduino follows the instruction correctly that fed by us. How can we send the instructions to the Arduino board? The instructions can be fed to the Arduino board by these tools, one is the Arduino programming language (based on Wiring), and the other one isArduino Software (IDE), based on Processing. For a long period of time Arduino has been the master brain for thousands of projects, from everyday objects to
Volume 02 Issue 07 July 2020

complicated scientific instruments. All kind of people around the worldwide like students, hobbyists, artists, programmers, and professionals can make use of this open-source software platform, so that it can be very helpful to the users. The Arduino UNO was founded by the Ivrea Interaction Design Institute as an easy tool with easy access for fast prototyping, targeted at students without a background in electronics and programming. Quickly it interacts the wider community, the Arduino UNO board started facing to adapt to new needs and difficulties as per the product. All the Arduino boards are full and fully open-source platform, helps the users to build them freely and clearly adapt them to their particular needs. The this software is very easy to access for all kind of users includes the beginers and the new learners, also flexible enough for advanced users. This software is applicable to all systems like Mac, Windows, and Linux. In the educational area the treachers and the students independently access this software. This paves the way to the technology development and new innovations. Other microcontrollers offer similar functionality. All of these things are taken the sufficient details of microcontroller programming and pack it up in an proper function for the use. Arduino UNO makes the working process simple with the microcontrollers:[5-7]

- Inexpensive -The Arduino UNO board is comparively low cost when compared to the other microcontrollers that available in the market.

- Cross-platform - The Arduino UNO Software (IDE) easily access all kind of the platforms like Windows, Macintosh OSX, and Linux operating systems. Most of the microcontrollers systems are limited to Windows.

- Simple programming platform - The Arduino Software (IDE) is very easy and simple for the beginners, available also for the advanced users to take advantage of as well. Also for the teaching staffs it is applicable to teach the students for the basic programming here.

- Open source and extensible software - The Arduino IDE software is typographed as open source tools, available for 
experienced programmers. This can access the $\mathrm{c}++$ libraries in the IDE software itself.

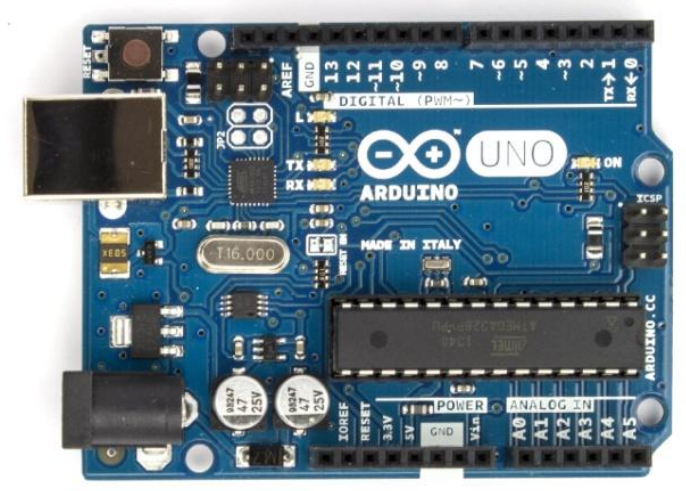

Figure.1: Arduino UNO

Specifications:

1 Microcontroller- ATmega328

2 Operating Voltage- 5V Input Voltage

3 Input Voltage -6 to $20 \mathrm{~V}$

4 Digital I/O Pins- 14Analog Input Pins :6

5 DC Current for the 3.3V Pin- $50 \mathrm{~mA}$

6 Flash Memory- $32 \mathrm{~KB}$ SRAM :2 KB

7 EEPROM- $1 \mathrm{~KB}$

8 Clock Speed- $16 \mathrm{MHz}$

General Pin functions:

1 LED: In that a LED is in-bulitedwith the digital pin 13. So that the differ in values causes the LED ON/OFF. It is simple in that pin the value is high the LED is in ON state and the value is low the LED in OFF state.

2 VIN: This Input Voltage pin is used to access the external power supply rather than connecting the USB port. So the additional power source is get access the board through this pin.

3 5V: This pin functions tosend as a $5 \mathrm{~V}$ as a output that passed through the regulator on the board. We can give supply to the board through the DC power source(7V-20V), also through USB port $(5 \mathrm{~V})$. If the voltage supplied through the $5 \mathrm{~V}$ or $3.3 \mathrm{~V}$, the board get damaged.

4 3V3: A 3.3 volt supply generated by the on-board regulator. Maximum current draw is $50 \mathrm{~mA}$.
5 GND: It is ground pin, used for grounding.

6 IOREF: Based on the microcontroller operations the voltage references provided by the board itself.

7 Reset: This reset pin is normally used to reset the program that stored in board.

8 Special Pin Functions: All the 14 Digital pins and the 6 Analog pins on the Arduino UNO board can be use as input/output. Each pin can operates at $5 \mathrm{~V}$. Based on the operating condition each pin can provide or receive as 20 $\mathrm{mA}$ and has an internal pull-up resistor (disconnected by default) of 20-50k ohm. The value does not exceeds 40 $\mathrm{mA}$ on any of the $\mathrm{I} / \mathrm{O}$ pins to avoid the permanent damage to the microcontroller. The Arduino Uno has 6 analog inputs, named A0-A5, which provide 10 bits of resolution (i.e. 1024 different values). Initially they measure from the ground to $5 \mathrm{~V}$, though it is possible to change the upper end of their range with the help of the AREF pin and the analogReference() function.

9 Serial: This pin specially functions for receiving and transmitting. The pins 0 (RX) and 1 (TX). It is used to receive (RX) and transmit (TX) TTL serial data. Pins of the ATmega8U2 USB-toTTL Serial chip are connected with these pins.

10 External Interrupts: The pins 2 and 3 are the external interrupts. These pins can be assigned to trigger an interrupt on a low value and a high value.

11 PWM(Pulse Width Modulation): The pins $3,5,6,9,10$, and 11 can provide 8 bit PWM output with the analog write function.

12 SPI(Serial Peripheral Interface): The pins 10 (SS), 11 (MOSI), 12 (MISO), 13 (SCK) supports the SPI communication by using the SPI library.

13 TWI(Two Wire Interface): The A4 or SDA pin and A5 or SCL pin supports TWI communication by using the wire library. 
14 AREF(Analog Reference): It is the Reference voltage for the analog inputs.

\subsection{Temperature Sensor:}

Temperature sensor senses the room temperature. This electronic device converts the data that sensed in the surrounding into the electronic data for recording purpose. There are many different types of temperature sensors.Here in this project we are using LM 35 temperature sensor. The LM35 temperature sensor is graded to work from $-55^{\circ}$ Centigrade to $150^{\circ}$ Centigrade with a udeviating scale factor of $+10 \mathrm{mv} /{ }^{\circ} \mathrm{C}$.. It is a tiny and low cost IC which can be used to measure temperature anywhere in the surrounding between $-55^{\circ} \mathrm{C}$ to $150^{\circ} \mathrm{C}$.

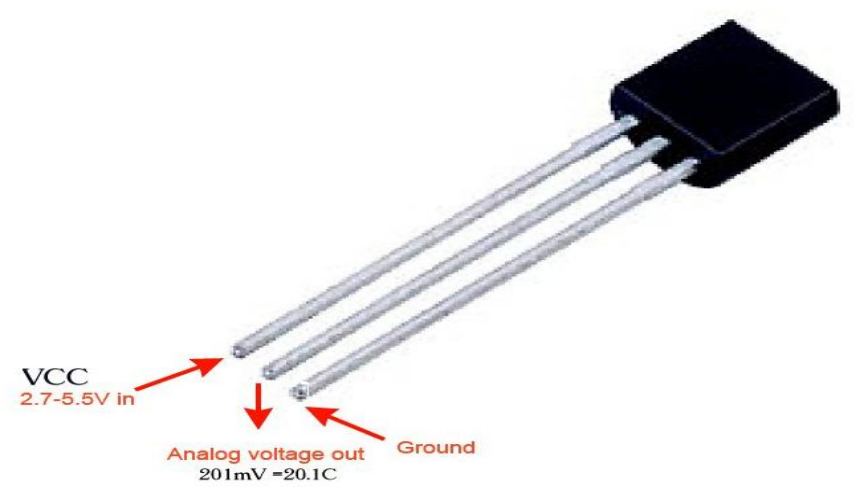

Figure.2: LM35 Temperature Sensor

Properties:

- Measures directly in degree Celsius (centigrade)

- Linear $+10.0 \mathrm{mV} /$ degree Celsius

- 0.5 degree Celsius accuracy (at +25 degree Celsius)

- Rated between -55 to +150 degree Celsius range

- Suitable for remote applications

- Low cost due to wafer-level trimming

- Operates from 4 to 30 volts

- Less than 60 Micro ampere current drains

- Low self-heating, 0.08 degree Celsius and Nonlinearity only +/- 1/4 degree Celsius

\subsection{Fan:}

It is an electronic appliance used to flow the air around its environment. We all know that fan contains blades, they act on the air. The Impeller, rotor, or runnerare the rotating assembly of blades.

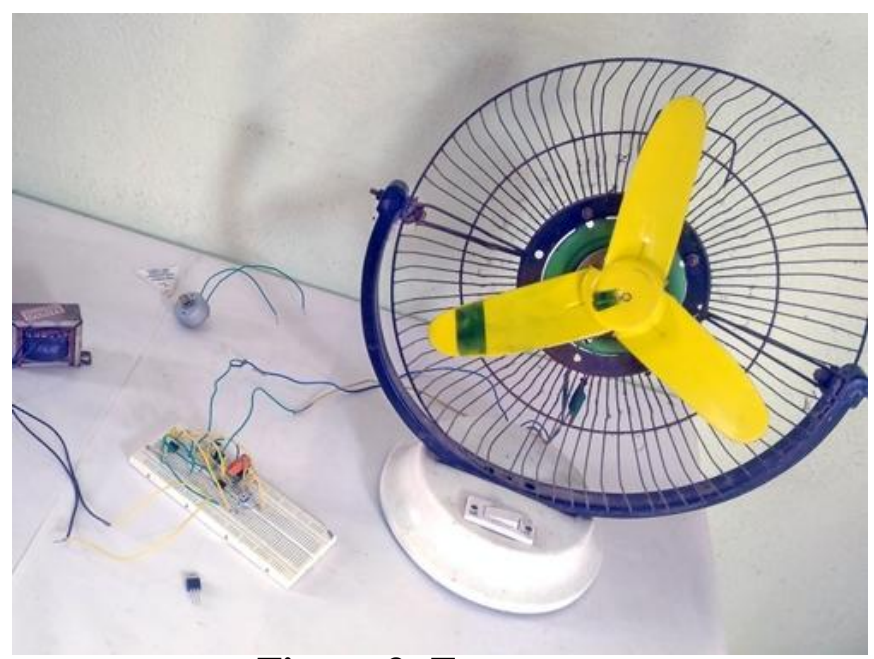

Figure.3: Fan

\subsection{LCD Display:}

Liquid crystal displays (LCDs) have found enormous success in the past couple of decades. They are used everywhere in our day to life. Some of the examples are from cellular phones, ebooks, GPS devices, computer monitors, and automotive displays to projectors and TVs to name a few. They play a critical role in the information age and are import elements of our daily life. Liquid crystals do not emit light. Their function is to modify the state of light produced by a light source in order to display images. The light is produced by either a direct backlight, which is placed directly beneath the liquid crystal panel, or edge light which is placed at the edge of a waveguide sheet. . Backlight is more suitable for large-size LCDs, because it can provide high light intensities, but it is bulky. Edge light is more suitable for small-size handheld LCDs, because it is compact, but its light output is limited. The common light sources for LCD lighting are cold cathode fluorescent lamps (CCFL), light emitting diodes (LED), external electrode fluorescent lamps (EEFL), and flat fluorescent lamps (FFL). CCFL consists of a glass tube with a cathode and an anode at the ends. The tube is filled with mercury gas. The inner surface of the tube is coated with a fluorescent (phosphor) material. When a voltage is applied across the two electrodes, some (primary) electrons are emitted by thermal motion in the cathode and accelerated toward the anode. There are also dichroic reflective polarizers, which have 


\section{www.rspsciencehub.com}

the advantage of high light efficiency. They pass incident light polarized in one direction and reflect incident light polarized in the orthogonal direction. The reflected light can be recycled by rotating its polarization into the direction of the transmission axis of the polarizers. The rotation of the polarization can be achieved either by a half waveplate or by a scattering medium.

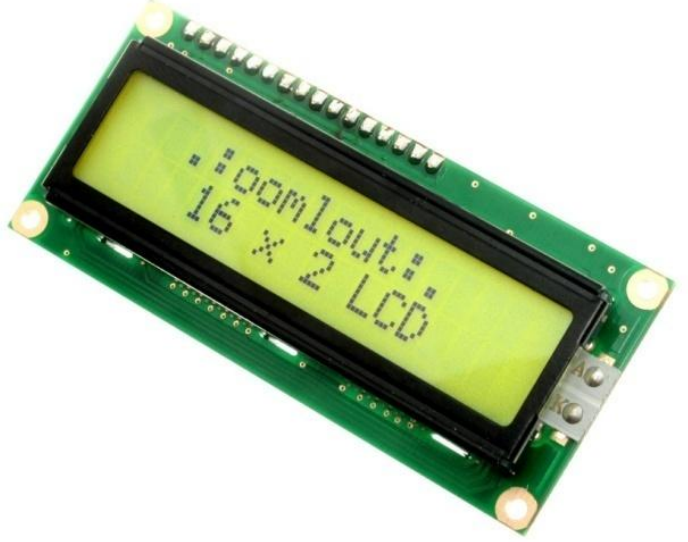

Figure.4: LCD Display

Pin Description

\begin{tabular}{|c|c|c|}
\hline $\begin{array}{l}\text { Pin } \\
\text { No }\end{array}$ & Function & Name \\
\hline 1 & Ground (OV) & Ground \\
\hline 2 & Supply voltage; $5 \mathrm{~V}(4.7 \mathrm{~V}-5.3 \mathrm{~V})$ & Vcc \\
\hline 3 & $\begin{array}{l}\text { Contrast adjustment; through a variable } \\
\text { resistor }\end{array}$ & $V_{E E}$ \\
\hline 4 & $\begin{array}{l}\text { Selects command register when low; and data } \\
\text { register when high }\end{array}$ & $\begin{array}{l}\text { Register } \\
\text { Select }\end{array}$ \\
\hline 5 & $\begin{array}{l}\text { Low to write to the register; High to read } \\
\text { from the register }\end{array}$ & Read/write \\
\hline 6 & $\begin{array}{l}\text { Sends data to data pins when a high to low } \\
\text { pulse is given }\end{array}$ & Enable \\
\hline 7 & \multirow{8}{*}{ 8-bit data pins } & $\mathrm{DB} 0$ \\
\hline 8 & & \begin{tabular}{|l|}
$\mathrm{DB} 1$ \\
\end{tabular} \\
\hline 9 & & \begin{tabular}{|l|l|} 
DB2 \\
\end{tabular} \\
\hline 10 & & \begin{tabular}{|l|l} 
DB3 \\
\end{tabular} \\
\hline 11 & & \begin{tabular}{|l|l|}
$\mathrm{DB} 4$ \\
\end{tabular} \\
\hline 12 & & \begin{tabular}{|l|l|}
$\mathrm{DB} 5$ \\
\end{tabular} \\
\hline 13 & & \begin{tabular}{|l|l|}
$\mathrm{DB} 6$ \\
\end{tabular} \\
\hline 14 & & DB7 \\
\hline 15 & Backlight $\mathrm{V}_{C C}(5 \mathrm{~V})$ & Ledt \\
\hline 16 & Backlight Ground (OV) & Led- \\
\hline
\end{tabular}

\section{Figure.5: Pin description}

\subsection{Potentiometer:}

The Potentiometer is an electric device that used to measure the Electro Motive Force of a given battery, the internal resistance of a cell. And also it is used to compare the EMFs of different cells. It can also use as a variable resistor in most of the applications.

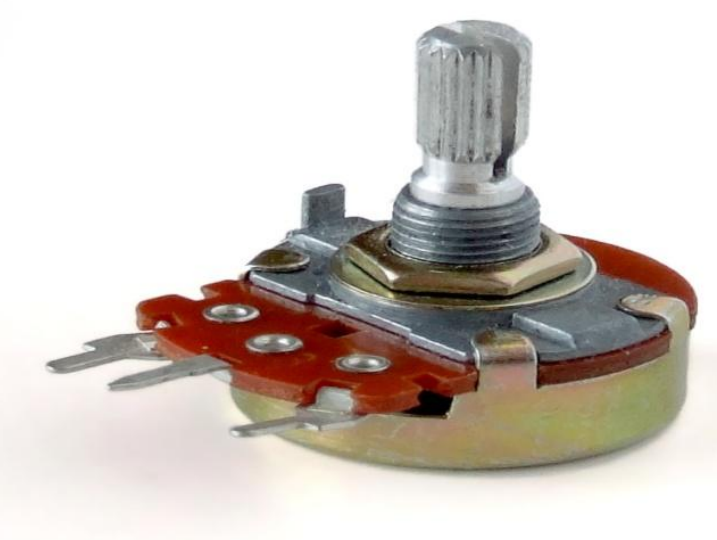

Figure.6: Potentiometer

\subsection{Transistor:}

A transistor is a semiconductor device used to amplify or switch electronic signals and electrical power. It is composed of semiconductor material usually with at least three terminals for connection to an external circuit.

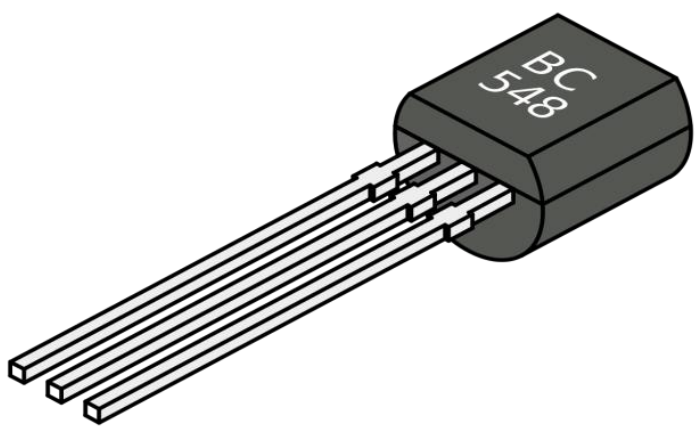

Figure.7: Transistor

\subsection{Resistor:}

Physical materials resist the flow of electrical current to some extent. Certain materials such as copper offer very low resistance to current flow, and hence they are called conductors. Other materials such as ceramic which offer extremely high resistance to the current flow are called as insulators. In electric and electronic circuits there is a need for materials with specific values of resistance in the range between that of a conductor and an insulator. These materials are called resistors and their values of resistance are 
expressed in ohms.

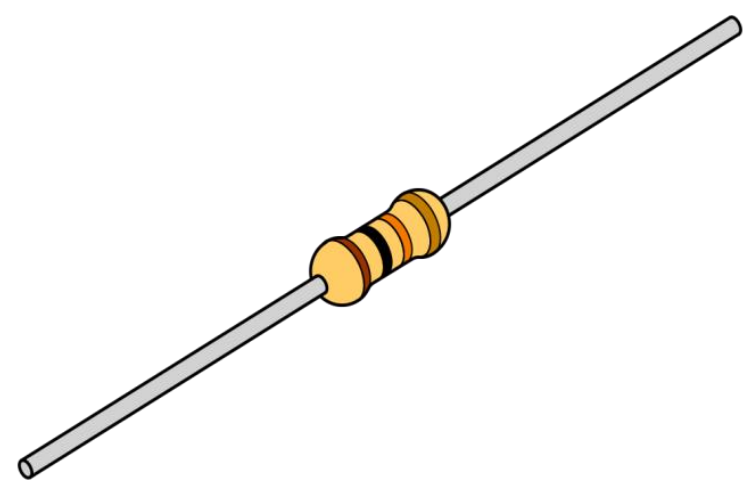

Figure.8: Resistor

\subsection{Relay:}

It is a switch which controls (open and close) circuits electromechanically. The main operation of this device is to make or break contact with the help of a signal without any human involvement in order to switch it ON or OFF. It is mainly used to control a high powered circuit using a low power signal.

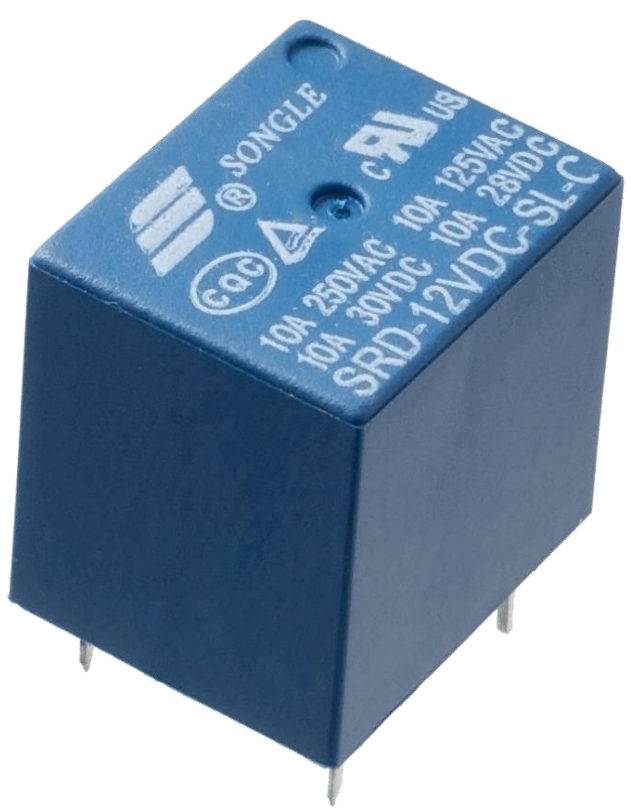

Figure.9: Relay

\subsection{Connecting wires:}

Connecting wires provide a medium to an electrical current so that they can travel from one point on a circuit to another. In the case of computers, wires are embedded into circuit boards to carry pulses of electricity.

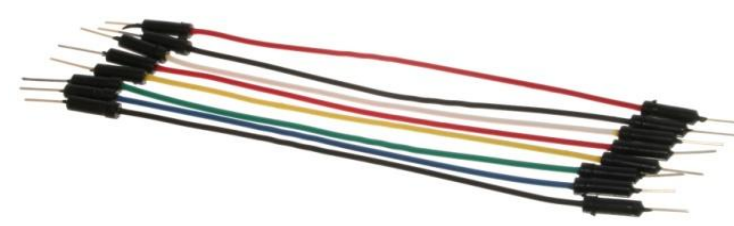

Figure.10: Connecting Wires

\section{Working:}

The working of this project is the ttemperature sensor LM35 senses the temperature and converts it into an electrical (analog) signal, which is applied to the ATmega328 microcontroller of the Arduino UNO Board. In this the Arduino UNO board converts the recorded signal from analog to digital signal. So that the recorded values of the temperature and speed of the fan are displayed on the LCD. When the temperature crosses $30^{\circ} \mathrm{C}$ the fan starts rotating.

A low-frequency pulse-width modulation (PWM) signal, whose duty cycle is varied to adjust the fan's speed is used. An inexpensive, single, small pass transistor-like $2 \mathrm{~N} 222$ or BD139 can be used here. It is efficient because the pass transistor is used as a switch.

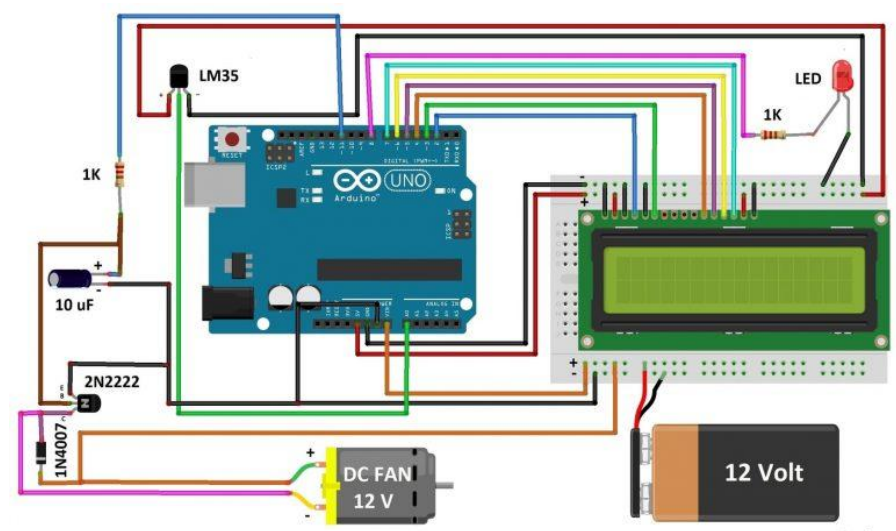

Figure.11: Circuit Diagram

Lm35 temperature sensor voltage to temperature conversion, now one of the most difficult thing is how to convert the voltage generated/output by the $\operatorname{lm} 35$ sensor at output in Celsius or Fahrenheit scales. Well this needs you to first go through the data sheet of temperature sensor and know about the characteristics of the sensor. 


\section{Program:}

\#include <LiquidCrystal.h>

LiquidCrystal lcd(2,3,4,5,6,7);

int Pin = A1; // the output pin of LM35

int $\mathrm{fn}=10$; // the pin where fan is

int $1 \mathrm{~d}=7$; // led pin

int tmp;

int tempMin $=24 ; / /$ the temperature to start the fan $0 \%$

int tempMax = 57; // the maximum temperature when fan is at $100 \%$

int fnSpeed;

int fnLCD;

void $\operatorname{setup}()\{$

pinMode(fn, OUTPUT);

pinMode(ld, OUTPUT);

pinMode(Pin, INPUT);

lcd.begin $(16,2)$;

Serial.begin(9600);

\}

void loop()

\{

tmp $=$ readTemp ()$; / /$ get the temperature

Serial.print( tmp );

if (tmp < tempMin) // if temp is lower than minimum temp

\{

fnSpeed $=0$; // fan is not spinning

analogWrite(fn, fnSpeed);

fnLCD $=0$;

digitalWrite(fn, LOW);

\}

if((tmp >= tempMin) \&\& (tmp $<=$ tempMax $)) / /$ if

temperature is higher than minimum temp

\{

fnSpeed $=$ tmp;//map(temp, tempMin, tempMax, $0,100)$; // the actual speed of fan//map(temp, tempMin, tempMax, 32, 255);

fnSpeed $=2 *$ fnSpeed;

fnLCD = map(tmp, tempMin, tempMax, 0, 100); //

speed of fan to display on LCD100

analogWrite(fn, fnSpeed); // spin the fan at the
fanSpeed speed

\}

if(tmp > tmpMax) // if temp is higher than tempMax

\{

digitalWrite(ld, HIGH); // turn on led

\}

else // else turn of led

\{

digitalWrite(ld, LOW);

\}

lcd.print("TEMP: ");

lcd.print(tmp); // display the temperature

lcd.print("C ");

lcd.setCursor(0,1); // move cursor to next line

lcd.print("FANS: ");

lcd.print(fnLCD); // display the fan speed

lcd.print("\%");

delay(200);

lcd.clear();

\}

int readTemp() \{ // get the temperature and convert it to celsius

tmp $=$ analogRead(Pin);

return tmp * 0.48828125 ;

\}

\section{Application:}

- Normally we use the regulator to change the speed of the fan. Here the room temperature changes the speed varies so that according to the temperature the fan rotates.

- The fan designed in this project, has the more scope to use in the Middle East countries. This product is more suitable for the hot regions.

\section{Conclusion \& Future Scopes:}

This project can be used in both the home and Industry. It helps in saving the energy and electricity. 
- To watch the environments that is not comfortable, or possible, for humans to monitor, especially for extended periods of time.

- Prevents waste of energy when it's not hot enough for a fan to be needed.

- To assist people who are disabled to adjust the fan speed automatically.

- In future case we can monitor more parameters like humidity, light and at the same time control them and also can send this data to a remote location using mobile or internet.

- Using this technology we can able to draw graphs of variations in these parameters using computer. And the temperature exceeds the limit; a call will be dialed to the respective given number by an automatic Dialer system.

\section{Reference:}

[1] Liu, Y., Zeng, J. and Wang, C. (2009). Temperature Monitoring in Laser Assisted Polymer Bonding for MEMS Packaging Using a thin Film Sensor Array, IEEE Sensors Applications Symposium, New Orleans, LA , USA.

[2] Jung, W.,You, J. and Won, S. (2008). Temperature Monitoring System for Inductive Heater Oven (pp.1734-1737), International Conference on Control, Automation and Systems, Seoul Korea.

[3] Chiueh, H., Choma, J. and Draper, J. (2000). Implementation of a temperature Monitoring Interface Circuit for Power PC Systems (pp.98-101), Proceedings of the 43rd Midwest Symposium on Circuits and Systems, Lansing MI, USA.

[4] Mehta V. K and Mehta R. (2007). Principle of Electronics, S.Chand \& Company Ltd.New Delhi, 438

[5] Theraja B. L and Theraja A. K. (2002). A Textbook of Electrical Technology, S. Chand \&Company Ltd. New Delhi, 2105
[6] Gayakwad R. A. (2004). Op-Amps and Linear Integrated Circuits, Pearson Edition (Singapore) Pte. Ltd., New Delhi, India, 315

[7] Mashud M. A. A, Barman S. C, Bhuiyan M. R. A, Islam Md.Serajul. (2013). Design and Implementation of Three Phase Unbalanced Voltage Motor Controller (pp.35-42), I.J. Intelligent Systems and Applications. 